\title{
Torsten Bultmann
}

\section{Die standortgerechte Dienstleistungshochschule}

Angeblich findet in der erweiterten Bundesrepublik wieder so etwas wie eine Hochschulreform statt. Tatsächlich war der administrativ-politische Aktivismus, der den Universitäten und Fachhochschulen gegenwärtig pausenlos Gesetzesnovellen, Richtlinien, Leistungskriterien, »Eckdaten« oder einfach nur schlaue Ratschläge verpaßt, seit mehr als 20 Jahren nicht mehr so intensiv wie heute. Die Begeisterung von Beteiligten und Betroffenen hält sich allerdings in Grenzen. Kein Zufall: Die den höheren Bildungsstätten überwiegend von außen angedienten »Innovationen « sind leicht als bloße »Sparpolitik $z u$ entlarven, wie sie gegenwärtig allenthalben stattfindet. Sämtliche Tätigkeiten an den Hochschulen sollen offenbar stärkeren ökonomischen Rationalitätskalkülen unterworfen werden, um unter Bedingungen stagnierender bis rückläufiger staatlicher Bildungshaushalte aus den chronisch unterfinanzierten Fachbereichen die letzten »Effizienzreserven« herauszupressen. Das ist jedoch nur die halbe Wahrheit.

\section{Das Verhältnis von Sparpolitik, Wissenschaftspolitik und Strukturmodernisierung - eine erste Annäherung}

Der Blickwinkel auf »Sparpolitik« suggeriert allzu leicht, das Hauptproblem wären konzept- und begriffslose Mittelkürzungen einer überforderten staatlichen Bildungsbürokratie. Der mit den aktuellen »Haushaltskonsolidierungen《 transportierte Diskurs »Alle müssen gemeinsam Opfer bringen! « ist jedoch bezogen auf die Hochschulen genauso falsch wie für andere gesellschaftliche Bereiche. Es gibt kein unpolitisches Sparen. Auch die gegenwärtige Magerkur für die Hochschulen ist mit zielgerichteten Konzentrations- und Differenzierungsprozessen verbunden, denen jeweils implizite wissenschaftspolitische Annahmen und Prioritätensetzungen zugrundeliegen. ${ }^{1}$ In den schlichten Worten des bayerischen Kultusministers

An der TU Berlin machen 7 Professoren in einem »Offenen Brief« die »extreme Überpolitisierung« für den heutigen Zustand verantwortlich (vgl. die Debatte in TU intern Nr. 1 und 2/1996). An der Carl von Ossietzky-Universität Oldenburg fordern 14 Professoren der Sprach- und Literaturwissenschaften (ebenfalls in einem »Offenen Brief«) als Reaktion auf die Ankündigung der Landesregierung, die Streichung der Slawistik zu erwägen, die radikale Einstellung der Lehrerausbildung (für Real-, Haupt- und Sonderschulen), die ursprünglich zum Kern des Oldenburger Reformkonzeptes gehörte (vgl. Uni-Info 6/96). 
Zehetmeier (Pressemitteilung 10.6.1996): »Die knappen Haushaltsmittel müssen dorthin fließen, wo sie am dringlichsten benötigt werden, nicht wo am lautesten gejammert wird.« Der strukturelle Zwang zur Unterscheidung von »wichtig « und »weniger wichtig« bzw. von Kern- und Randaufgaben der Hochschulen fühtt nun geradewegs dazu, daß die tradierte Machtverteilung innerhalb des Wissenschaftsapparates noch gestärkt wird.

Anders ausgedrückt: Die »ideologische Form« des Sparens besteht darin, »das institutionell Übliche zum Standard zu erheben bzw. zu verkehren...\& (Fried, Kaindl, Markard 1995, 162). So werden etwa Frauenforschungsprojekte gestrichen oder es geraten die Sozial- und Geisteswissenschaften an Technischen Universitäten unter besonderen Legitimations- und Kostensenkungsdruck. Ein ebenso konsequenter Ausdruck aktuellen Gesundschrumpfens ist die »Zusammenlegung« von Parallelinstituten, die infolge von »Sezessionen « in den frühen 70er Jahren entstanden sind, welche sich aus konkurrierenden Wissenschaftskonzepten und Ansätzen der Studienreform ergaben. ${ }^{2}$ Die gesamte aktuelle Hochschulentwicklung wäre daraufhin zu befragen, inwieweit sich Entpolitisierung, Entdifferenzierung, kurz: Nivellierung und Altemativenarmut, sowie zunehmende Außensteuerung gegenseitig bedingen. Die Fragestellung ist allein deswegen produktiv und provokativ, weil »Autonomie« und »Differenzierung« gerade ideologische Leitbegriffe der gegenwärtig einflußreichsten hochschulpolitischen ThinkTanks sind (vgl. Stifterverband 1994, Müller-Böling 1995).

Verkürzt wäre nun allerdings die Schlußfolgerung, es ginge unter dem Deckmantel von »Sparpolitik « und »Kosteneffizienz« allein um die Beseitigung kritischer Wissenschaft im Sinne eines konservativen Roll-Backs, wiewohl einzelne Effekte in dieser Richtung sicher keineswegs uner-

Die Beispiele sind beliebig. Sie drücken etwas von der aktuellen Kultur an deutschen Hochschulen aus. Es ist ein (partiell unappetitlich anmutendes) Hauen und Stechen jeder gegen jeden ausgebrochen.

2 Fried, Kaindl und Markard (1995) haben diese Vorgänge für die Psychologie an der FU Berlin analysiert. Aktuell wird offenbar mit einem ähnlichen Regiebuch die Fusion der (noch vorhandenen) zwei juristischen Fachbereiche an der Universität Hamburg vorbereitet. 1974 wurde dort neben der traditionellen (Standes) Juristenausbildung ein Reformstudiengang $\gg$ Jura II $\ll$ mit sozialwissenschaftlicher Akzentsetzung eingerichtet. Die Begründung für die Zusammenlegung in der Hamburger Universitätszeitung ist von überregionaler Bedeutung, weil bundesweit austauschbar: »Die angesichts der Sparmaßnahmen künftig verfügbaren Stellen reichten nicht aus, um in Zukunft zwei getrennte Fachbereiche beizubehalten, die gegenüber anderen juristischen Fakultäten und Fachbereichen funktions- und konkurtenzfähig wären« (Uni-HH 1/1996). War ursprünglich die Unterscheidung von traditionellen akademischen Standards - in unserem Fallbeispiel: von »den anderen juristischen Fakultäten« - ein zentraler Impuls für Reformexperimente ist heute diese Differenz ein fiskalisches Argument für das exakte Gegenteil: für ihre Beerdigung. Unser (durchaus willkürlich gewähltes) Beispiel lehrt, wie durch bloßen Kostendruck hochschulinterne Entscheidungen quasi automatisch durch eine Außenperspektive geprägt werden, deren Maßstab der traditionelle Ausbildungs- und Arbeitsmarkt ist. 
wünscht sind. Die bildungsbürgerliche Ordinarienuniversität alten Stils ist jedoch unvereinbar mit der etwa im Bundesbericht Forschung 1996 (BMBF 1996, 6f) beschriebenen Leitlinie einer integrierten Entwicklungsdynamik von »Bildung «, »Wissenschaft« und »Wirtschaft « als entscheidender politischer Achse der »Informationsgesellschaft«. Seit längerem schon verstärkt sich der Druck auf die Hochschulen, unter Bedingungen knapper Staatsfinanzen ihre Leistungsangebote »bewertbarer« zu machen oder: anstatt nur über fehlende Mittel zu jammern, sich besser intern selbst zu optimieren. Offenbar werden in der gegenwärtigen Situation die Weichen für eine »nachhaltige Organisationsreform « (Hans-Jürgen Ewers) gestellt, welche die traditionellen technokratischen Steuerungsmechanismen der Wissenschaftsplanung, wie sie als Zusammenwirken von Staat, Wirtschaft, Hochschulgremien und Standesverbänden im wesentlichen als juristisch geronnene Kompromisse aus den Konflikten der 70er Jahre entstanden sind, schrittweise durch neue Elemente wettbewerbsförmiger Regulierung wissenschaftlicher Arbeitsabläufe ersetzt.

Innerhalb der staatlichen Planungsgremien von Bund und Ländern hat sich im Konsens mit führenden Wissenschaftsverbänden offenbar ein größtes gemeinsames Vielfaches an Überlegungen für unabdingbare Neuansätze herausgebildet, welches sich nach Behrens $(1996,126)$ in zwei Punkten zusammenfassen läßt:

* das »das Hochschulrecht beherrschende Kollegialprinzip« (ebd.) - anders gesagt: die pauschalen Standesprivilegien der Professorenschaft - wird als Strukturmerkmal in seiner effektiven Steuerungswirksamkeit für wissenschaftliche Abläufe ebenso infrage gestellt wie das Prinzip der Gruppenuniversität;

* angestrebt wird »eine Verlagerung von Entscheidungskompetenzen vom Staat auf die Hochschulen« (ebd.), die als zielgerichtete Deregulierung allerdings unmittelbar ineinandergreifen soll mit einer Aufwertung des Hochschulmanagements und einer Stärkung der Hochschulleitungsstrukturen auf der Ebene von Rektoren/Präsidenten und Dekanen,

Ausschlaggebend für diese Überlegungen ist ganz offenkundig die Annäherung der Hochschulverfassung an ein Modell privater Unternehmen. Dies wird allein daran deutlich, daß sich die Stärkung der $\gg L$ Leitungsebenen« an der Übertragung von Direktionsrechten mißt, die denen eines betrieblichen Managements immer ähnlicher werden.

Gemeinsamer Nenner derartiger Maßnahmen ist die Entkoppelung der Strukturfrage von der Frage nach den Aufgaben einer Hochschule. Dabei kann eine professionelle operative Trennung von akademisch-politischen und Verwaltungsaufgaben durchaus sinnvoll sein, wenn gleichzeitig beide Handlungsstränge in ein spezifisches Verhältnis zueinander gesetzt werden. Aktuell laufen diese Vorgänge jedoch überwiegend auf eine Entpolitisierung der Steuerungs- und Entscheidungsproblematik zugunsten inhaltslos-funktionalistischer »Effizienzsteigerung «, kurz: Kostensenkung plus Output-Maximierung, hinaus. Dabei entziehen sich entsprechende De- 
regulierungsmaßnahmen zunächst einer unmittelbaren politischen Bewertung, sie knüpfen an Teilwahrheiten und Schein-Evidenzen einer blokkierten Hochschulentwicklung an und können sich etwa - durchaus ambivalent - gegen »konservativ-ständische« und »demokratisch-partizipatorische« Funktionsmerkmale gleichermaßen richten. Die nordrhein-westfälische Ministerin für Wissenschaft und Forschung, Anke Brunn, bringt die von ihr angestrebte »Funktionalreform « gar auf den Dreiklang »Mehr Autonomie in der Hochschule - weniger Regeln durch den Staat - stärkere hochschulinterne Demokratie« (MWuF NRW 1995, 5). ${ }^{3}$

Unabhängig davon, was von diesem Versprechen zu halten ist, soll durch diesen Hinweis zumindest angedeutet werden, daß aus den aktuellen Entwicklungsblockaden des Hochschulsystems verschiedene politische Optionen begründbar sind, die bis in die, zum Teil in sich widersprüchlichen, symbolischen Integrationsbemühungen der staatlichen Ebene abfärben. Problematisiert wird zum einen generell die Ineffizienz des $»$ Gremienwesens« insofern es (in den heutigen Schwundformen der Gruppenuniversität) auf einer Repräsentation von verschiedenen Interessen beruht. Nachhaltig öffentlich in Frage gestellt ist aber auch die für die deutsche Hochschulgeschichte konstitutive Institution des Professoriats (Daxner 1991, 53f), welches auf der impliziten Gleichsetzung von Forschungsspezialisierung und wissenschaftsplanerischer Kompetenz, bzw. von »Amtsautorität und Sachautorität« (Daxner ebd., 57; Nitsch 1991, 19ff) beruht.

Der Auslöser für den hektischen hochschulpolitischen Aktivitätsschub der staatlichen Planungsbürokratie seit Anfang der 90er Jahre ist zweifellos das Motiv ökonomisch-technischer Effizienzsteigerung im Sinne der »Standortfrage«. Welche Politik sich daraus entwickelt, ist allerdings in Nuancen beeinflußbar. Dies hängt auch davon $a b$, welche Definition der »Krise der Hochschulen《 sich in der Öffentlichkeit durchsetzt. Handelt es sich vor allem um die Problematik fehlender Ressourcen im Sinne einer (technischen) Überlastung des Systems? Handelt es sich um ein $»$ Effizienzproblem《 historisch überlebter Strukturen? Handelt es sich um ein Politik- und Demokratiedefizit in der Regulierung wissenschaftlicher Abläufe? Oder um eine spezifische Komposition verschiedener dieser Momente? Eine genaue Bewertung ist abhängig von einer Interpretation der Hochschulexpansionsphase und ihrer Resultate, der wir uns nun zuwenden müssen.

3 Ein Vorschlag des von Anke Brunn geleiteten Gesprächskreises »Funktionalreform« beinhaltet etwa, daß die Aufgabe des Dekans auch von einem Vier-Personen-Rat, der sich nach dem Grundsatz der Viertelparität zusammensetzt, wahrgenommen werden könnte. (MWuF NRW 1995, 83) 


\section{Von der Expansion zur Überlast - von der Überlast zur Dauerlast - von der Dauerlast zum "Effizienzproblem《}

»Humboldt ist in der Masse erstickt! «, so diagnostizierte der Präsident der Hochschulrektorenkonferenz (HRK), Erichsen, auf der HRK-Jahresversammlung 1992 in Rostock - und prägte ein mittlerweile geflügeltes Wort. Dem liegt offenbar eine Anschauung zugrunde, es hätte so etwas wie ein tradiertes und bewährtes Modell der deutschen Universität gegeben, welches man versäumte, an die neuen Realitäten der Massenhochschule infolge der sozialen Öffnung höherer Bildungswege ab Mitte der 60er Jahre anzupassen. Der Wissenschaftsrat, das einflußreichste Gremium der Wissenschaftsplanung im vorparlamentarischen Raum, postuliert folglich in seinen aktuellen 10 Thesen zur Hochschulpolitik $(1993,18)$ das neue Leitbild seine(r) Anpassung der Hochschule an den institutionellen Typus 'Dienstleistungsbetrieb'« in ausdrücklicher Gegenüberstellung zum traditionellen Humboldtschen Typus.

Bei näherer Betrachtung ist der anfänglich scheinbar klare Satz, Humboldt sei in der Masse erstickt, ausgesprochen mehrdeutig, weil die darin enthaltene Entgegensetzung der Kriterien »Wissenschaftlichkeit« und »Masse« nicht weiter begründet wird. Ist damit lediglich die organisatorisch-technische Schwierigkeit beschrieben, unter Bedingungen chronischer Unterfinanzierung der Hochschulen ein angemessenes wissenschaftliches Bildungsniveau garantieren zu können? Oder handelt es sich um die unbelegbaren ideologischen Behauptungen konservativer Elitetheorien, die »natürlichen« Begabungsreserven in der Bevölkerung seien ungleich verteilt, wissenschaftliche »Befähigung« würde nur eine kleine Minderheit auszeichnen? $?^{5}$ Die Spannweite zwischen beiden Fragen - welche wahrscheinlich auch das Kompromißspektrum innerhalb einer Organisation wie der

4 Im einzelnen heißt es dort (S.19): $\rightsquigarrow$ Damit stehen traditionelle Grundsätze der deutschen Universität zur Disposition: Einheit von Forschung und Lehre für alle Universitätsmitglieder, Verbindung von Forschung und Lehre für jeden Universitätslehrer, Freiheit von Studium und Lehre, Kollegialität und Selbstverwaltung.« Der Wissenschaftsrat ist seit 1957 ein Organ der Bund-Länder-Wissenschaftsplanung in Kooperation mit den führenden Wissenschaftsverbänden. Ihm gehören zum einen Abgesandte aller zuständigen Ministerien (politische Kommission), vor allem jedoch hochkarätige »ernannte« Professoren an, auf die sich HRK, Deutsche Forschungsgemeinschaft (DFG), Max-Planck-Gesellschaft (MPG) und Arbeitsgemeinschaft der Großforschungseinrichtungen (AGF) im Konsens verständigt haben (Wissenschaftskommission). Daraus ergibt sich das »spezifische Gewicht« der jeweiligen »Empfehlungen« (vgl. Bultmann 1993, 27f).

5 So äußert sich etwa der Augsburger Ordinarius (em.) Finkenstaedt in der Zeitschrift des Hochschulverbandes zur Sache (»Studierfähigkeit heute《): »Die besten drei Prozent eines Jahrgangs überleben in jedem Bildungssystem. Eine brauchbare allgemeine Studierfähigkeit ist vermutlich unter bestimmten Bedingungen für fünfzehn Prozent erreichbar. Weitere Prozente - und wir werden sehr viel mehr Prozent brauchen - können durch eine fachgebundene Hochschulzulassung versorgt werden« (Forschung \& Lehre 10/95, 564). Begründet wird dieses darwinistische Szenario nicht weiter. Man weiß es halt! 
HRK erfaßt - beschreibt die interessenpolitisch repräsentierten Positionen zur Hochschulreform.

Zunächst war die Bildungsreform, einschließlich der Modernisierung und Expansion der Hochschulen, eine der erfolgreicheren »großen« Sozialreformen, die real Lebensbedingungen in der gesellschaftlichen Breite verbessert hat. Die Zahl der Schulabgänger mit (allgemeiner und fachgebundener) Hochschulreife stieg von 11,3\% pro Altersjahrgang im Jahre 1970 auf 37,2\% (1994) (BMBF, Grund- und Strukturdaten 1995/96, 85). Die Studierendenzahlen aller Hochschultypen kletterten von 510 Tsd. 1970 auf 1,85 Mio. im Jahre 1994 (ebd., 141). Quantitativ wurde damit lediglich eine internationale Angleichung vollzogen: in allen Industriestaaten lag die Hochschulzugangsquote pro Altersjahrgang in den 80er Jahren zwischen über $20 \%$ bis z.T. mehr als $50 \%$ (USA) (vgl. Teichler 1990, 29). Bis Mitte der 70er Jahre ging diese Öffnung »höherer « Bildungswege hierzulande mit einem extensiven Ausbau der Hochschulen durch Erweiterungen, Neugründungen und einer analogen Vermehrung von Verwaltungs- und Wissenschaftspersonal einher. Dieser Prozeß war flankiert von einem Ausbau staatlicher Planungs- und Lenkungsfunktionen für den gesamten Wissenschafts- und Bildungsbereich, der quasi in das System wachstumsorientierter etatistischer Globalsteuerung integriert wurde. Diese Instrumentarien haben sich im wesentlichen bis heute erhalten und sind zum Objekt unterschiedlich inspirierter »Bürokratismuskritik« geworden, der man ihren jeweiligen demokratischen oder reaktionären Charakter nicht sofort ansieht. Es ist häufig zutreffend betont und analysiert worden, daß die wesentlichen Impulse der Hochschulexpansion den damaligen Erfordernissen kapitalistischer Modernisierung entsprachen. Die Mobilisierung ökonomisch-technischer Innovationsressourcen aus den Hochschulen war über die Mechanismen der traditionellen Eliteuniversität ebensowenig möglich wie die von der Industrie damals dringend nachgefragte quantitative Vermehrung des akademischen Fachkräftepotentials (»Humankapital «). So gesehen war der Ausbau der Staatsfunktionen zwangsläufig. Um nur ein Beispiel zu nennen: Ende der 60er Jahre weigerten sich etwa die etablierten Fakultäten, Informatik und Biochemie als selbständige Fächer anzuerkennen. Während in den USA dafür längst eigene Ausbildungsprogramme existier-

6 In einer Resolution der Arbeitsgemeinschaft der Vorsitzenden der Fakultätentage Deutschlands (AVF) heißt es etwa: »In Wahrheit werden die Universitäten fortschreitend von einer kollegial verfaßten, insbesondere wissenschaftlicher Erkenntnis dienenden Körperschaft in eine hierarchisch-bürokratisch organisierte Ausbildungseinrichtung verwandelt.« Die Motive des Staates werden in den (so wörtlich) 》Gravamina« der Resolution auf solche der kleinlichen Kontrolle und der Durchsetzung »berufspraktischer« Bezüge reduziert; insbesondere einer Bewertung von »Lehrerfolgen« wird der Kampf angesagt. Als ideale Gegenwelt schimmert die Rückkehr zur »reinen« Wissenschaft und zum ordinarialen $\gg$ Fachvertretungsprinzip« auf (Forschung \& Lehre 12/1995, 690f). 
ten, wurden diese Bereiche hier der mathematischen bzw. chemischen Fakultät untergeordnet. Ihre Entfaltung als separate Fächer war nur dadurch möglich, daß die Länderministerien entsprechende Prüfungsordnungen durchdrückten (vgl. Reuhl 1992, 100).

Im staatlichen Handeln der Hochschulreformperiode lassen sich ökonomische und soziale »Motive« nicht strikt auseinanderhalten. Die »soziale Öffnung « der Hochschule war in der politischen Öffentlichkeit einerseits demokratietheoretisch inspiriert (»Bildung als Bürgerrecht!《), sie war ebenso gleichbedeutend mit einer wirtschaftlich unumgänglichen Erschließung neuer Bildungsreserven aus sozialen Schichten, denen bis dahin diese Möglichkeit verschlossen geblieben war. Als Bedingung dafür galt die Entkoppelung von individuellen Studienkosten und Elterneinkommen. Darauf zielten die zeitgleiche Abschaffung von Studiengebühren (1970) in der Form von Hörergeldern und die Einführung des BAFöG als Instrument sozialstaatlicher Bildungswerbung (mit einer anfänglichen Gefördertenquote von über $40 \%$; heute: $20 \%$ ). Daß aktuell beides wieder auf der politischen Agenda steht, d.h. daß emsthaft über die Einführung von Studiengebühren diskutiert und ein (wachsender) Darlehensanteil des BAföG als individuelle Beteiligung an den Ausbildungskosten privat verzinst wird, markiert das endgültige Finale dieser politischen Konstellation von Anfang der 70er Jahre.

Die zunehmende Relativierung der Formen staatlicher Verantwortung erstreckt sich auch auf andere bürokratische Hinterlassenschaften der Reformperiode: als bildungsökonomisch-arbeitsmarktpolitisch agierender Akteur mußte der Staat damals etwa auf die Normierung und Vergleichbarkeit von akademischen Qualifikationsstandards über die Regulierung von Prüfungen, Studienordnungen und die relative Angleichung von Studienbedingungen einwirken. Ambivalent war dies insofern, als es einerseits die Nivellierung individueller Differenzen in repressiver und kontrollierender Form bedeutete, die als Moment »systemkonformer Qualifikationsplanung« eine entsprechende Kritik radikaldemokratischer Hochschulreformer (vgl. Becker/Jungblut 1972) erfuhr; andrerseits führte diese bürokratische Vereinheitlichung zu einer relativen Gleichheit und Gleichwertigkeit der Hochschulen und wirkte folglich Bestrebungen entgegen, das Hochschulsystem nach angelsächsischem Vorbild stärker intem hierarchisch zu differenzieren. Unter heutigen Bedingungen eines gesättigten akademischen Arbeitsmarktes bei gleichzeitig verschärfter Konkurrenz um »Leistung « und »Qualität« werden diese politischen Relikte vor allem als Deregulierungsdefizit bewertet. Exemplarisch dafür die Beschwerde des Generalsekretärs des Stifterverbandes für die Deutsche Wissenschaft, Horst Niemeyer, in der Zeitschrift des Hochschulverbandes:

»Jede Universität wird gleich behandelt, um die Gleichheit der Lebensverhältnisse herzustellen und die Studentenströme gleichmäßig zu verteilen. (.....) Die Interessen staatlicher Bürokratie und 
akademischer Mittelmäßigkeit ergänzen sich hier mittlerweile mühelos, weil sie Gleichheit der Lebensverhältnisse auch in der gelehrten Welt wünschen.« (Forschung \& Lehre 6/1996, 292)

Die Konsequenz aus dieser Kritik ist die stärkere elitäre Gliederung des Hochschulsystems in der Spannweite zwischen akademischen Berufsfachschulen ganz unten und Forschungskollegs der Spitzenklasse ganz oben, was als mehr oder weniger ausgeprägtes Orientierungsprofil mittlerweile in allen relevanten hochschulpolitischen Entwürfen von HRK, Wissenschaftsrat, Kultusministern und Bund-Länder-Planungsgremien Verhandlungsgegenstand ist (Bultmann 1993, 12f). Eine passende Festvortrags-Philosophie dafür gibt es inzwischen auch: »Exzellenz in Forschung und Ausbildung ist ein höherer Wert als die Homogenität des Systems, das sie schafft« (Mittelstraß 1992, 46).

Wirken die Resultate der Hochschulexpansionsphase auf diese Weise als unentschiedene Konflikte bis heute nach, war diese Expansion im engeren Sinne eigentlich schon Mitte der 70er Jahre mit Einbruch der ökonomischen Strukturkrise beendet. Die weiter steigende Nachfrage nach Hochschulbildung mündete nicht mehr in eine analoge Entwicklung des Beschäftigungssektors (vgl. Hartung 1993, 411). Wachsende Akademikerarbeitslosigkeit war eine für die damalige BRD relativ neue Erscheinung: sie vervierfachte sich zwischen 1975-1985, während die allgemeine Arbeitslosigkeit im gleichen Zeitraum »nur « um das 2,2fache stieg (ebd.). Seinen Ausdruck fand dieser Sachverhalt in der Politikerphrase der »Entkoppelung von Bildung und Berechtigung «. Die politische Form der Beendigung des Hochschulausbaus war der berühmte $»$ Doppelbeschluß« der Ministerpräsidenten, Kultus- und Finanzminister aus dem Jahre 1977, die Hochschulen weiter für alle Studierwilligen offenzuhalten, aber ihre Finanzen einzufrieren. Begründet wurde dies als zeitlich befristetes $\gg$ Überlastprogramm《, da fälschlicherweise von einem demographisch bedingten Rückgang der Studierendenzahlen (»Untertunnelung des Studentenberges «) ausgegangen wurde (vgl. Bultmann 1993, 19). Selbst dieser Öffnungsbeschluß war noch ein »Kompromiß«: die politische Linke kritisierte daran mit einiger Plausibilität, daß sich der Staat unter Bedingungen juristischer Beibehaltung des Ziels »soziale Öffnung der Hochschulen« aus seiner finanziellen bildungspolitischen Verantwortung schrittweise zurückziehen würde. Der Anteil der Hochschulausgaben am Bruttosozialprodukt ist tatsächlich von 1,3\% Mitte der 70er Jahre auf 0,9\% zu Beginn der 90er Jahre gesunken (HRK 1996, 18). Konservative Vertreter des Wissenschaftssystems beanstandeten vor allem die mangelnde Konsequenz des Beschlusses $^{7}$ als Ausdruck politischer Feigheit, welche davor zurückschrecken wür-

7 So erst kürzlich der Wissenschaftsminister des Freistaates Sachsen (Meyer 1996,6): »Ein klassisches Beispiel für einen solchen falschen Kompromiß ist für mich der Öffnungsbeschluß von 1977: Wer die Hochschulen mit Studenten vollstopft, auf deren Eingangs- 
de, endlich die überfälligen Maßnahmen sowohl einer stärkeren Selektion beim Hochschulzugang als auch einer internen Differenzierung des Hochschulsystems nach »Leistungsgesichtspunkten « durchzusetzen.

Dieses durch den »Öffnungsbeschluß« fixierte unentschiedene bildungspolitische Kräfteverhältnis wirkt nun schon annähernd 20 Jahre. Bekannt ist, daß die »Überlast《 zur »Normallast « wurde. Seit diesem Zeitpunkt haben sich die Studierendenzahlen annähernd verdoppelt, Ausstattungsbedingungen und Stellensockel blieben hingegen im wesentlichen konstant. Die Betreuungsrelationen (Verhältnis Studierendenanzahl pro Stelle wissenschaftliches Personal) verschlechterten sich an den Universitäten der alten Länder (ohne Medizin) von 13 (1975) auf 24 (1994), an den Fachhochschulen gar von 16 auf 40 im gleichen Zeitraum (HRK 1996, 9). Die GEW hat errechnet, daß der geringfügige Zuwachs der wissenschaftlichen Stellen an Universitäten um ca 10\% vor allem durch den Ausbau der medizinischen Einrichtungen absorbiert wurde (GEW 1994, 19f).

Diese Quantifizierung der Überlast ist einerseits aufschlußreich, jedoch nicht hinreichend, in gewissem Sinne sogar irreführend, für die Bestimmung ihrer qualitativen Auswirkungen und systemdeformierenden Effekte bis in die inneren Strukturen wissenschaftlicher Arbeitsabläufe hinein. Von einer statistisch suggerierten gleichmäßigen Belastung aller Hochschulangehörigen kann nämlich keine Rede sein. Die absolute Zahl der als wissenschaftliches Personal an Universitäten (alte Länder) statistisch erfaßten Personen hat sich im Verhältnis zu einem konstanten bzw. nur geringfügig gestiegenen Stellensockel von ca 70 Tsd. 1978 auf 107 Tsd. 1993 vermehrt. Die Anteil der Professoren blieb darunter von 1979 bis 1993 im wesentlichen zwischen 20 Tsd. und 21. Tsd. konstant (BMBF, Grund- und Strukturdaten 1995/96, 236). Die Personalvermehrung bei stagnierenden Haushaltsstellen hat folglich vor allem in der Personalkategorie »wissenschaftliche Mitarbeiter« in entsprechend zunehmend befristeten und zerstückelten Arbeitsverhältnissen (vgl. Enders 1989) stattgefunden. Nimmt man die gesamte Hochschulexpansionsphase als Maßstab, dann haben sich die Professoren in den letzten dreißig Jahren vervierfacht (ein Vorgang, der allerdings schon Ende der 70er Jahre abgeschlossen war), die nichtprofessoralen Wissenschaftler hingegen verneunfacht (Enders 1995, 11). Die Überlast wurde so, insbesondere in den letzten 10 Jahren, einerseits durch einen Zuwachs an prekären Beschäftigungsverhältnissen über reguläre Haushaltsmittel, andrerseits durch eine Steigerung von Drittmittelstellen überwiegend in Form von Privatdienstverträgen (Wissenschaftsrat 1995a, 28) »aufgefangen«. Dies hat »die Schere zwischen der Kürze der

qualität die Fachbereiche und Fakultäten keinen Einfluß haben, darf sich nicht wundern, wenn das System blockiert wird.k 
befristeten Verträge und der Länge der Qualifizierungsphasen« des sog. wissenschaftlichen Nachwuchses weiter auseinanderklappen lassen (Enders 1995, 12). Unterschiedliche Befragungen (ebd., 11) haben zu Schätzungen geführt, daß mittlerweile $60-80 \%$ der Regelaufgaben der Hochschulen in Forschung und Lehre von dieser Personengruppe ausgeführt werden. ${ }^{8}$ Die Chancen auf eine Dauerbeschäftigung sind aber bei einer konstant bleibenden Zahl von Professorenstellen umgekehrt proportional zur quantitativen Expansion der Mittelbauangehörigen gesunken. Dies gilt auch unter Zugrundelegung der Annahme, daß nur ein Teil davon eine Berufung anstrebt, die mittlerweile zur annähernd einzigen Chance für ein »reguläres« Beschäftigungsverhältnis als Hochschulwissenschaftler/-in geworden ist.

Der Oldenburger Unipräsident, Michael Daxner, bezeichnet dies als »die unsinnigste Personalkostenverschwendungsmaschine, die es gibt: Wir bilden Leute für Millionen Mark aus, die wir dann mit 40 Jahren hinausschmeißen, wenn sie am meisten leisten könnten. Auf der anderen Seite verbeamten wir Leute ohne jede Leistungsprognose mit 30 Jahren, und die bleiben 35 Jahre im Dienst« (Die ZEIT 26.4.96; vgl. auch Daxner 1993, 170ff). Auch aus einem marktwirtschaftlichen Blickwinkel ist dies irrational. Die absurde »Außeralltäglichkeit« (Preißer 1994) einer solchen innerwissenschaftlichen Arbeitsteilung wird deutlich, wenn man diese Realität mit den sich in den letzten Jahren häufenden Schlagzeilen von einer »drohenden Professorenlücke« konfrontiert, was offenbar nichts mit einer analogen »Nachwuchslücke« zu tun haben kann: In den nächsten zehn Jahren werden mehr als $50 \%$ der Professorenstellen altersbedingt frei. Diese »Pensionierungswelle« ist eine direkte Folge des »Öffnungsbeschlusses« von 1977, der zu einer abrupten Stagnation der Planstellen - und zwar nach einer vorhergegangenen »Berufungswelle« während der Hochschulexpansionsphase - geführt hat. Aktuell drückt sich dies in der zunehmenden Überalterung der höchsten und systemprägenden Hierarchiestufe der deutschen Hochschulen aus (Wissenschaftsrat 1995, 10f). Dieses Mißverhältnis der sozialen Generationenfolge verschlechtert nicht nur die Chancen der jeweils Nachrückenden, es befördert auch Tendenzen der Stagnation und der paradigmatischen Verkrustung im wissenschaftlichen Erkenntnispro-

8 Eine empirische Befragung des Kölner Max-Planck-Instituts für Gesellschaftsforschung (1990/91) hinsichtlich des Zeitbudgets von Professoren widerlegt die von diesen und ihren Standesvertretungen häufig geäußerte Standardklage, es bliebe in der Massenuniversität immer weniger Zeit für die Forschung. Im Durchschnit trifft dies offenbar nicht zu. Der entsprechende Zeitaufwand hat sich im Vergleich mit Befragungen von 1976/77 and 1983/84 nicht signifikant verringert (Schimank 1994). - Es kann hier natürlich nicht um die Suche nach "Sündenböcken « gehen; eine moralisierende Personifizierung der Hochschulkrise verhindert nur die Erkenntnis ihrer Ursachen. Das Problem des Professorenstandes ist nicht primär die subjektive Verfaßtheit einer Personengruppe, sondern die (historisch akkumulierte) Überkomplexität der Berufsrolle (Becker/Wehling 1993, 79). 
zeß (Bochow/Joas 1987, 14). Das Problem besteht nun auf absehbare Zeit darin, daß die berufungsfähige »Reserve« gemessen an der Zahl der Habilitationen die zunehmenden Stellenvakanzen - natürlich bei extremen Unterschieden zwischen den einzelnen Fachgebieten - kaum wird decken können, wie sich an den Grunddaten des Wissenschaftsrates (1995) zum Personalbestand der Hochschulen unschwer ablesen läßt. Die Gesamtzahl der Habilitationen entspricht zwar in etwa den wiederzubesetzenden Stellen. Eine planwirtschaftliche Zuteilung im Verhältnis 1:1 ist jedoch nicht nur aufgrund der Inkompatibilitäi der verschiedenen Fachgebiete unmöglich, sie würde auch den für das deutsche Hochschulsystem konstitutiven Grundgedanken einer »Auswahl der Besten« ad absurdum führen (GEW 1995, 33). Spätestens hier wird die Sache peinlich.

Es wird zudem deutlich, daß es sich um ein »hausgemachtes « Problem des vorherrschenden Wissenschaftsbegriffes handelt, welches in keinem unmittelbaren Kausalitätsverhältnis zur Unterfinanzierung der Hochschulen steht - und »im Prinzip« sofort behoben werden könnte: vereinfacht gesagt, durch Abschaffung der Habilitation und eine Professionalisierung wissenschaftlicher Berufsausübung. Im Grunde genommen gibt es an Hochschulen nur einen anerkannten wissenschaftlichen Beruf, nämlich den des Professors. Der gesamte sog. Mittelbau befindet sich in einer nicht klar definierbaren Zwitterstellung zwischen den ihm gestellten Qualifizierungsauftrag (Promotion, Habilitation), der Funktion als disponibler Selektionsreserve und eigenverantwortlich durchgeführten Tätigkeiten in Forschung, Lehre und Dienstleistungen, ohne die das Hochschulsystem längst zusammengebrochen wäre. Die gesamte Personalstruktur ordnet sich dabei in arbeits- und statusrechtlicher Hinsicht der auf die Grundlagenforschung und das ideale Leitbild der $\gg$ Gesamtvertretung eines Faches in Forschung und Lehre« ausgerichteten Professur unter. Dieses Bild einer zunftförmigen Gemeinschaft von Meistern und Gesellen - mit der Habilitation als individuellem »Meisterstück « - entspricht zudem immer weniger der funktionalen Differenzierung wissenschaftlicher Arbeit in Forschung, Lehre, Beratungstätigkeit, Wissenschaftsmanagement und -transfer.

9 Die Zahl der jährlich abgeschlossenen Promotionen hat sich von 11.300 (1970) auf 20.038 (1992, alte Länder) etwa verdoppelt. Die Zahl der jährlichen Habilitationen stagnierte während der ganzen 80er Jahre um die 1000, sie ist bis 1993 geringfügig auf 1319 (neue Länder: 109) gestiegen (GEW 1995, 53-57). Aufgabenwachstum der Hochschulen und Attraktivität des Professorenamtes haben sich offenbar umgekehrt proportional entwickeit. Das ist insofern kein Zufall, als die Habilitation beruflich eine Sackgasse ist, fur die Altemativen außerhalb der Universitätslaufbahn nicht existieren. Bei nicht gelingender Berufung sind die Betreffenden für den Arbeitsmarkt sowohl zu alt als auch »überqualifiziert.«

10 Die Bundervereinigung akademischer Mittelbau (BAM) weist ständig darauf hin, daß die horizontal differenzierte Aufgabenexpansion der Hochschulen, kurz: die Arbeit, die konkret erledigt werden muß, kaum noch etwas mit der tradierten Personalstruktur bzw. mit der Unterscheidung zwischen Professoren und »Nachwuchs« zu tun hat: »In den Hoch- 
Die Problematik ist nicht neu. Schon das legendäre SDS-Gutachten $\gg$ Hochschule in der Demokratie« wies auf den Widerspruch zwischen der zunehmend erforderlichen betriebsförmigen Rationalität wissenschaftlicher Arbeitsabläufe und der »überhöhten Amtsautorität des Lehstuhlinhabers《 hin (Nitsch/Gerhardt/Offe/Preuß 1965, 143). Daß dieses Problem heute noch existiert, ist eine politisch zu verantwortende Meisterleistung, ein Ausdruck der Tatsache, daß die Hochschulreformen auf dem Weg einer halbierten Demokratisierung und Rationalisierung steckengebliebenen sind; noch präziser: sie sind zum Teil »nach hinten « korrigiert worden, indem etwa alle hochschulpolitischen Gesetzeswerke ab Mitte der 70er Jahre die gremienpolitische und statusrechtliche Position der Professoren wieder gestärkt haben (ausführlich: Plander 1986).

Heute scheint ein Punkt erreicht zu sein, daß diese Strukturen halbdemokratisch-technokratisch-oligarchischer Kompromisse, welche die hiesige Hochschullandschaft regulieren, aus sich selbst, d.h. aus dem Kernbereich der sie prägenden Elite kaum noch reproduktionsfähig - ergo auch nicht mehr legitimationsfähig - sind. Ohne folglich aus sich heraus eine Alternative präsentieren zu können, ist das »System《 sturmreif geworden für marktwirtschaftliche Modernisierungsdiksurse und -praktiken. Die Überlast kann umdefiniert werden in ein »Effizienzproblem《. In dem Maße, wie dabei die Perspektive auf ein betriebswirtschaftlich-funktionalistisches Problemarrangement verengt wird, gerät die wirkliche Effizienzreserve einer aufgabenbezogenen arbeitsteiligen Professionalisierung und Demokratisierung wissenschaftlicher Tätigkeit aus dem Blick.

\section{Professoren als Dienstleister, Studierende als Kunden: die »wirtschaftlich denkende Hochschule «}

Aus einer betriebswirtschaftlichen Außenperspektive betrachtet erscheint das alltägliche Tun und Treiben an einer durchschnittlichen deutschen Hochschule in der Tat als abenteuerlich: desorganisierte Überkomplexität, völlige Disparatheit von Interessen, selbstempfundenen Aufträgen und Handlungszielen. Von daher ist es plausibel, den Hochschulen vor allem ein »Managementdefizit《 zu diagnostizieren, wenn man unter Management rein funktionalistisch die optimale Koordination von knappen Ressourcen auf definierte Ziele hin versteht. Alle Unternehmensberatungsfirmen kommen daher mehr oder weniger zu austauschbaren Ergebnissen (Daxner 1993, 127).

schulen haben sich in allen organisatorischen Einheiten und auf allen Ebenen spezifische Teilaufgaben ausdifferenziert. Beispiele dafür sind Leistungen für Forschung, Lehre und Studium, Wissenschaftsmanagement, Wissens- und Technologietransfer sowie wissenschaftliche Weiterbildung, Hochschulplanung und Hochschuldidaktik« (BAM 1991, 59). Die herkömmlichen Personalhierarchien stehen dabei einer reflektierten Professionalisierung dieser Aufgaben entgegen. 
Die dynamischen Berater, die Anfang der 90er Jahre alle in den Startlöchern standen und auf Großaufträge warteten, weil sie offenbar angesichts der staatlicherseits angezettelten »Effizienzdebatte« ein riesiges Geschäft erhofften, hatten ein entscheidendes Defizit: Ihnen fehlte die Sensibilität für wissenschaftskulturelle Besonderheiten und die spezifische Befindlichkeit der im Hochschulbereich organisierten Interessen. Diese Lücke konnte - ideologiepolitisch bisher sehr effektiv - durch das 1994 gegründete Centrum für Hochschulentwicklung (CHE), in gemeinsamer Trägerschaft von Hochschulrektorenkonferenz und Bertelsmann-Stiftung mit Sitz in Gütersloh, insbesondere durch die mediale Omni-Präsenz seines Geschäftsführers Detlef Müller-Böling, zuvor Rektor der Universität Dortmund, geschlossen werden. Der Erfolg besteht allein darin, daß sich mittlerweile alle hochschulpolitischen Akteure am CHE abarbeiten müssen, welches die Öffentlichkeit am laufenden Band mit Kongressen, Tagungen und Positionspapieren zu allen für entscheidend gehaltenen Fragen von »Studiengebühren« bis $»$ Abiturreform« versorgt. Den Modellversuch »Finanzautonomie« des niedersächsischen Ministeriums für Wissenschaft und Kultur (Pressemeldung 9.6.95) begleitet etwa ein Beirat unter Vorsitz des CHE. In Peter Glotz hat das Centrum darüberhinaus einen prominenten Promoter seiner strukturpolitischen Innovationsvorschläge gefunden.

Müller-Böling $(1994,272)$ geht von folgendem Befund aus: "Angesichts der bürokratischen Regelungswut und der Vielfalt von Reformansätzen ist kein einheitliches Bild der Hochschulen mehr auszumachen. Während man sich in Einzelmaßnahmen erschöpft, fehlt eine gesamtheitliche Vision für eine Hochschule der Zukunft. « Dieser Befund, bei dem Unternehmensberater im Regelfall stehen bleiben, wird nun scheinbar historisch »aufgelöst«. Alle Akteure, welche auf der Ebene staatlichen Handelns oder organisierter Interessen die Steuerung der Hochschulen mittelbar oder unmittelbar beeinflussen würden, gingen von völlig unterschiedlichen, tendenziell unvereinbaren, Leitbildern des Zwecks einer Hochschule aus, die auch jeweils unterschiedliche Konsequenzen für Entscheidungsstrukturen und Regulierung der Finanzströme hätten. Hier sei der Kern des Problems zu sehen. Idealtypisch vereinfacht handelt es sich um vier verschiedene Leitbilder:

Die Gelehrtenrepublik: Die Hochschule würde als lockere Gemeinschaft ausgewiesener Forscher verstanden, die individuell ihre akademische Freiheit verwirklichen und die so gewonnenen Erkenntnisse sin einem eher unstrukturierten Kommunikationsprozeß« (ebd.) an die Studierenden weitergeben. Dabei erfolge die Finanzierung nach dem »Alimentationsprinzip« (ebd.), d.h. indem die Gelehrten ihre nicht hinterfragbaren Forderungen von der Gesellschaft erfüllt sehen wollen. Anders gesagt: Die Ordinarienuniversität.

Die (nachgeordnete) Behörde: Dies entspräche der Behandlung der Hochschulen als staatliche Einrichtungen, die über Dienst- und Haushaltsrecht 
oder Besoldung etatistisch gesteuert würden, woraus eine analog zur Hochschulexpansion gewachsene Wissenschaftsadministration wiederum ihre Legitimation zöge. Akademische Strukturen seien demgegenüber von nachgeordneter Bedeutung. Finanziert würden Kapazitäten über den Input, d.h. die vermutete bzw. zu erwartende Leistung (ebd. 273).

Die Gruppeninstitution: Dies wäre die Hochschule als Ort der institutionalisierten Austragung von Interessengegensätzen, die jeweils »mit Hilfe demokratischer Mechanismen ausgeglichen bzw. geschützt werden müssen« (ebd.). Hochschulpolitische Entscheidungen und Finanzallokationen würden nicht nach einem »Gesamtinteresse « sondern vor allem nach ihrem jeweiligen gruppenbezogenen Nutzen beurteilt. Nach Müller-Böling wohnt der Gruppenhochschule offenbar ein spezifischer Mechanismus inne, daß sich ständig neue, repräsentiert sein wollende Interessenbereiche ausdifferenzieren: "Zunehmend entwickeln sich 'Minderheitsvertretungen', gesetzlich vorgeschrieben bereits als Behinderten- und Frauenbeauftragte, selbstorganisiert als Ausländerbeauftragte, Schwulen- und Lesbenreferate o.ä...« (ebd.), was nach dieser Logik einer weiteren Verflüchtigung des »Gesamtinteresses « Vorschub leisten würde. Der Unterschied zwischen vielen konkreten Interessen und »Gesamtinteresse « ist nicht benannt (man kann ihn allerdings plastisch erahnen!); in jedem Fall erscheinen spezifisch organisierte Interessen als das völlig Jenseitige und Abseitige der »Wissenschaft $\ll$.

Der Dienstleistungsbetrieb: Nach dieser Vorstellung ist die jeweilige Hochschule »Produzent von Dienstleistungen im Bereich von Forschung und Lehre, von Transfer, Wirtschaftsförderung und Kultur« (ebd., 274), also von Angeboten, für die sie kein Monopol hat, sondern von vornherein in Konkurrenz zu anderen Anbietern auf dem Gebiet der (staatlichen oder privaten) Berufsausbildung oder hochschulfreien bzw. industriellen Forschung steht. Dem entspräche eine »Optimierung der Input-Output-Relation « (ebd.), welche in letzter Konsequenz nur über eine marktförmige Regulierung dieser »Produktion « zu erreichen sei. Institutionalisierte Entscheidungsprozesse würden aus diesem Blickwinkel vor allem unter dem Aspekt ihrer möglichen Verringerung und zeitlichen Verkürzung betrachtet.

Dieser Diagnose ist eine gewisse Raffinesse und Plausibilität nicht abzusprechen, weil sie tatsächlich die Realität konkurrierender Steuerungsimpulse im Hochschulbereich bis zu einem gewissen Grade abbildet, anders gesagt: die Kompromißbreite technokratischer Regelungen, deren divergierende Zielsetzungen sich in allen Hochschulgesetzen wiederfinden. MüllerBöling entscheidet sich nun keineswegs für ein bestimmtes dieser »Modelle«. Er spricht eher allen eine relative Legitimität zu und konstruiert lediglich den »Rahmen einer Vision« für die Hochschule der Zukunft als quasi »offenes Projekt« aus einem Bündel von Merkmalen (»profiliert«, »wettbewerblich «, »wissenschaftlich«, »autonom《, »wirtschaftlich $\ll)$, die den 
jeweils unterschiedlichen Traditionen und Interessen entnommen zu sein scheinen (ebd., 275). In der Praxis des CHE entspricht dem eine kluge Politik der Erprobung »wechselnder Allianzen«; vereinfacht gesagt: mal mit Mittelbau und Studierenden gegen die »uneingeschränkte(n) Individualrechte« der Professoren (Müller-Böling 1985, 4), mal mit konservativen Standesorganisationen gegen Studierende und Schüler, wie beispielsweise beim Plädoyer für eine stärkere Selektion des Hochschulzugangs (»Leipziger Erklärung«) auf einer gemeinsamen Tagung von CHE und Sächsischem Wissenschaftsministerium im Juni 1995 (vgl. Bultmann/ Weitkamp 1995). Dieses Changieren ist vor allem einer Strategie der Akzeptanzbeschaffung geschuldet und verdeckt, daß die Hauptrichtung des Laborierens an den Hochschulen durch Orientierung am Typus »Dienstleistungsbetrieb « bestimmt ist, nicht nur in den CHE-Konzepten sondern in der gesamten Wissenschaftsadministration.

Deren Kritik an der festgefahrenen Entwicklung läßt sich so zusammenfassen, daß das deutsche Hochschulsystem niemals Steuerungsmechanismen hervorgebracht hat, die auf einer Bewertbarkeit von Leistungen und einer entsprechenden 》leistungsgesteuerten Ressourcenverteilung《 (Wissenschaftsrat 1993, 18) beruhen. Die in den 70er Jahren eingespielten Mechanismen einer kapazitätswirksamen Input-Finanzierung, die ihre komplizierten Zuteilungsschlüssel im wesentlichen an der Nachfrage nach Studienplätzen orientiert, hätten gerade eine bis heute reformresistente »Tonnenideologie« (Block 1994, 220) erzeugt. Anders gesagt: Installiert wurde ein ritualisierter Verhandlungsmechanismus zwischen Staat und Hochschulen, bei dem diese auf jeden Aufgabenzuwachs, etwa durch ein Wachstum der Studierendenzahlen, nicht mit einer aufgabenadäquaten Veränderung ihrer inneren Strukturen, sondern mit der Forderung nach »mehr Geld« reagierten. Aus diesem Blickwinkel würde jede Zusatzfinanzierung als Belohnung von forcierten Fehlentwicklungen erscheinen.

Als Dreh- und Angelpunkt einer »nachhaltigen« Veränderung der Hochschulstrukturen kristallisiert sich folglich das Gelingen des Übergangs von einer Input-Finanzierung zu einer solchen, die sich an Leistungsindikatoren des »Outputs« mißt, heraus:

»Die wirtschaftliche und wirtschaftlich denkende Hochschule ist selbstverständlich nicht auf Gewinnerzielung ausgerichtet...." - ein Differenzkriterium ist folglich das Verhältnis von Profit- und Non-Profit-Management - $\gg .$. Sie sollte aber sehr wohl eine Optimierung der Zweck-Mittel-Relation anstreben. Zu der Input-Betrachtung, die bisheriges (Haushalts-) Verhalten prägt, muß eine Beurteilung des Outputs im Sinne einer individuellen und gesellschaftlichen Bewertung der Leistung treten.« (Müller-Böling 1985, 11)

Müßig zu erwähnen, daß hier mit »gesellschaftlicher Bewertung« keine Erprobung neuer Partizipationsmodelle gemeint ist, sondern lediglich eine Dokumentierbarkeit der »Kosten« in Relation zu meßbaren »Ergebnissen«. 
Eine derartige Transparenz ist nicht per se abzulehnen. Im Gegenteil, dies müßte gerade auch der Anspruch einer demokratischen Öffentlichkeit sein. Daxner $(1993,127)$ hat recht, wenn er Kritikern der Haushaltsglobalisierung, die mit dem Totschlagargument kommen, »Qualitäi sei nicht »quantifizierbar « (was im Grunde eine logisch widersinnige Aussage ist), entgegenhält, daß Kennzahlen auch ein sozialwissenschaftliches Instrument sein können, »um Entscheidungen zu rationalisieren, und nicht um sie zu ersetzen.« Auch emanzipatorische Zielsetzungen können und müssen quantifiziert werden. Ohne politisch abrechenbare Quotierungsindikatoren würde sich Gleichstellungspolilitk etwa nur in leeren Bekenntnissen erschöpfen. Allerdings haben wir aktuell keine offene Entscheidungssituation, in der herrschaftsfrei über die Anwendbarkeit theoretischer oder politischer Referenzkriterien für quantitative Daten verhandelt würde. In dem Maße, wie eine formale Quantifizierung von Arbeitsabläufen eingebettet ist in eine verschärfte Konkurrenz von in ihren Startpositionen ungleichen Akteuren um immer knappere Mittel, verstärkt sich die Tendenz einer inhaltsleeren Beschleunigungsstrategie.

Die Schlüsselfrage bei alldem ist: Wie kann man wissenschaftliche Erträge »bewerten«? Die aktuell dominante Art der Beantwortung dieser Frage ist gleichbedeutend mit dem zielgerichteten »Verschweigen « ihres politischen Kerns: die Ergebnisse wissenschaftlicher Anstrengungen werden nicht in Relation zu einem definierbaren gesellschaftlichen Nutzen legitimiert, sondern vor allem »gezählt« und konkurrenzförmig tabelliert. Darin wurzelt die ausgreifende Ranking- und Quantifizierungsmanie, die das Hochschulsystem verstärkt seit Anfang der 90er Jahre heimsucht. Hochkomplexe Vorgänge wie »Erkennen《 oder »Lernen« werden auf quantifizierbare Größen reduziert - was allerdings ein »qualitativer « Vorgang ist! Zählen und Proportionieren läßt sich in Abhängigkeit vom Erkenntnisziel dann so ziemlich alles: vom Seitenumfang der Publikationen pro Stelle wissenschaftliches Personal, über die Lufthansa-Kilometer pro Lehrdeputatsstunde (vorrangig bei C 4 - Ost) bis zur »Kontakthäufikeit nach Hierarchieebenen «, mit der aus der Zahl der formalen Kommunikationsakte von Hochschulangehörigen, aufgeschlüsselt nach Statusgruppen, vermeintlich Rückschlüsse auf die »Organisationskultur« einer Universität gezogen werden können (Sporn 1992, 133f).

Bei dieser Vielfalt von Möglichkeiten zeichnet sich jedoch in den hochschulpolitischen Planungsetagen eine gewissen Verständigung dahingehend $a b$, daß »vertraute « Leistungsparameter, die traditionellen professoralen Distinktionsbedürfnissen entsprechen (Zahl del »Schüler«, Zahl der Veröffentlichungen) aufgegriffen und erweitert und zusätzlich um Mechanismen einer Effizienzoptimierung der Lehre ergänzt werden (vgl. etwa: Wissenschaftsrat 1993, 56f; »Eckwertepapier« 1993). Regelmäßig genann- 
te Richtgrößen einer Indikatorsteuerung sind etwa: Durchschnittstudienzeit, Zahl der erfolgreichen Absolventen pro Fachbereich, Zahl der Promotionen, Habilitationen oder (sonstigen) Publikationen, Anzahl der Sonderforschungsbereiche, der Graduiertenkollegs, Umfang der Drittmittel pro Fachbereich oder pro Stelle wissenschaftliches Personal etc.

In der Praxis sieht die Sache so aus, daß von den zuständigen Länderministerien wachsende Anteile der allgemeinen Grundausstattungsmittel der Hochschulen in speziellen Fonds reserviert und nach derartigen Leistungskriterien vergeben werden. "Dies ist verbunden mit einer schrittweisen Deregulierung des Haushaltsvollzugs (vgl. Behrens 1996), was bereits rein funktional betrachtet »starke« Entscheidungsträger auf Hochschulebene erforderlich macht. In diesem Zusammenhang wird schließlich deutlich, aus welcher Logik heraus am Anfang des aktuellen hochschulpolitischen Aktivismus die Politisierung der (vermeintlich zu langen) »Studienzeit《 stand, und zwar völlig ungeachtet der Tatsache, daß bisher ein Zusammenhang zwischen der Dauer des Studiums und der beruflich-fachlichen Relevanz des Abschlusses nicht nachgewiesen werden konnte (Daxner/Schindler 1993, 178). Zum einen ist dies sicher auf Motive populistischer Vereinfachung und symbolischen Problemmanagements zurückzuführen (ebd., 177). Die Thematik eröffnet die Möglichkeit wechselnder Personalisierungsstrategien: mal ist die für defizitär erklärte »Qualität der Lehre« auf dem Sündenkonto der Professoren im Visier, mal kann, je nach Anlaß, die »Studierfähigkeit« der Studierenden bezweifelt werden, um die Erprobung selektiverer Hochschulzugangsregularien $\mathrm{zu}$ begründen (Stichwort: »Wechselnde Allianzen $($ ). Gleichzeitig jedoch ist die »Studienzeit« eine geeignete Variable für eine stärkere ökonomische Bewertung und durch diese zunehmend gesteuerte Reorganisation der gesamten Hochschulabläufe. Wenn ich Verschiedenes und Unvergleichliches - etwa die Vielfalt an Fachtraditionen, Fachkombinationen und individuellen Studienmotivationen - gegenseitig vergleichbar machen will, brauche ich ein einheitliches Maß. Das einzige ökonomische Maß ist die Zeit. Die Durchsetzung eines rigiden Zeitregimes erstreckt sich auf den gesamten wissenschaftlichen Arbeitsproze $ß$, wenn etwa einerseits studentisches Verhalten entsprechend reguliert wird, gleichzeitig aber auch Ausstattungsmittel der Fachbereiche für Forschung und Lehre zunehmend nach studienzeitbezogenen Kriterien (Absolventenzahl, Durchschnittsstudiendauer) zugewiesen oder reduziert werden (vgl. etwa Wissenschaftsrat 1993, 581). »Studienzeit《 - oder die in Zeitquanten ausgedrückte Kombination von Studieninhalten - würde inner-

11 Beispiel Nordrhein-Westfalen: 1994 wurden 10\% der staatlichen Mittel nach Absolventenzahlen vergeben ( $90 \%$ in herkömmlicher Form); 1995 verdoppelte sich der Outputbezogene Anteil auf 20\%; davon 10\% nach Vergabekriterien wie 1994 und weitere 10\% nach sog. forschungsbezogenen Kriterien (Drittmittel, Promotionen). 
halb dieses ökonomischen Mechanismus vor allen Dingen unter dem Aspekt des »Zeitgewinns « für die Forschung bzw. der finanziell relevanten Vermehrung ihrer Ergebnisse erscheinen (soviel zum Thema »Qualität der Lehre«!). Auf diese Weise entstünde ein sich selbst verstärkendes $\gg$ wettbewerbliches« Leistungsverhalten. Letzte Konsequenz wäre die Realisierung eines primitiv-technokratischen Wachstumsmodells innerhalb der Hochschule, bei dem wissenschaftlicher »Fortschritt« mit der bloßen Anzahl faktenförmig isolierter »Ergebnisse« und der Geschwindigkeit ihrer »Produktion« gleichgesetzt würde.

Noch handelt es sich allerdings um Entwicklungstendenzen, die erst rudimentär ausgebildet sind. Es hat wenig mit Flexibilisierung und (ökonomischer) Haushaltsautonomie zu tun - eher mit dem Gegenteil -, wenn eine Indikatorsteuerung über rechenschaftspflichtige Eckdaten der jeweiligen Landesregierung inszeniert wird, als deren Administratoren dann die sog. »herausgehobenen Entscheidungsträger « auf Hochschulebene erscheinen (vgl. Franke 1994, 39). Ohne eine Flexibilisierung des Personalbereiches wird es auch keine »nachhaltige« Deregulierung hochschulinterner Prozesse geben, wenn man sich etwa vor Augen führt, daß über $70 \%$ der Hochschulausgaben auf diesen Bereich entfallen und entsprechend beamtenund tarifrechtlich gebunden sind (Behrens 1996, 124). Aber diese Probleme sind durchaus erkannt: mittlerweile spricht sich auch die HRK (1996, 27) für befristete Professuren und Teilzeitarbeitsverhältnisse für diese Gruppe aus; ergänzt um Elemente leistungsabhängiger Besoldung. Daß die Professorenschaft in ihrer überlieferten Form ein Auslaufmodell ist, kann bei allen aktuellen Ungewißheiten als gesicherte Annahme gelten. Allerdings erfolgt der Denkmalssturz diesmal aus rechtsliberaler Stoßrichtung.

Für den Übergang von einem Regime punktuell-interventionistischer Sanktionen und Begünstigungen, in dem sich »leistungsorientierte « Ressourcensteuerung aktuell noch erschöpft, zu verstärkten Formen (formaler) ökonomischer Selbstregulierung, ist die aktuell entbrannte - und daher so bald nicht verlöschende - Diskussion über die Einführung von Studiengebühren von strategischer Bedeutung. Es käme einer Täuschung gleich, Studiengebühren lediglich unter dem Aspekt der Hochschulfinanzierung als Kompensation für fehlende staatliche Mittel zu betrachten. Es geht vor allen Dingen um die effektive Verkoppelung von individuellem Bildungsverhalten, Hochschulressourcen und gesellschaftlichen Märkten:

»Denn Fehlverhalten einzelner Hochschulen und Hochschullehrer würden sich unmittelbar 'auszahlen'. Es käme zu einem Wettbewerb um Studierende, der über konkurrenzfähige Studiengangprofile und attraktive Studienbedingungen geführt würde."(Müller-Böling 1985a, 6) ${ }^{12}$

12 In einer Denkschrift des Kronberger Kreises (1993, 18), einer Vereinigung monetaristischer VWL-Professoren, heißt es bündiger zum Thema »Studiengebühren«: »Die studen- 
Anders gesagt: Die Studierenden müßten etwa die künftige $»$ Rendite « ihrer Studiengebühren schärfer kalkulieren und sich damit zwangsläufig stärker am Arbeitsmarkt und an eingeschliffenen gesellschaftlichen Karrieremustern orientieren; dieser Mechanismus wird noch verstärkt, wenn, wovon ausgegangen werden kann, die Mehrheit von ihnen Studiengebühren nur über kreditförmige Mechanismen der Vorfinanzierung, quasi als Vorgriff auf künftige Einkommen, wird aufbringen können. Von seiten der Fachbereiche würde folgender Komplementäreffekt einsetzen: in dem Maße, wie ihre materielle Ausstattung zunehmend auf Einnahmen aus Studiengebühren angewiesen und darüberhinaus die staatlichen Grundmittel absolventenbezogen »differenziert« wären, müßten sie attraktive Angebote zur Anwerbung von Studierenden entwickeln und sich auf diese Weise ebenso auf den Markt beziehen, da diese »Attraktivität« in direkter Relation zu den gesellschaftlichen Chancen der jeweiligen Studienabschlüsse stünde. Dies ist gleichbedeutend mit der Positionierung der Studierenden als Kunden, wie dies im neuen Wissenschaftszweig des »Universitätsmarketing « auch als methodischer Ansatz gepflegt wird: $» D i e$ Student/inn/en werden hier weniger als Mitglieder, sondern als Zielgruppe der universitären Maßnahmen gesehen« (Sporn 1992, 10). Diese »Kundenposition «, deren Kehrseite die Aufhebung der Steuerungsfunktion des - in seiner institutionalisierten Struktur fraglos reformbedürftigen - politischen Repräsentationsprinzips der Gruppenuniversität ist, mit einem Zuwachs an hochschulpolitischem Einfluß gleichzusetzen (Müller-Böling 1985a, 6), erscheint allerdings zynisch.

Die »wirtschaftlich denkende Hochschule« ist eine offenkundige Analogiebildung zur »atmenden Fabrik« aus der Lean-Production-Terminologie. Ideologiepolitisch besonders geschickt ist es, derartige Hochschulsteuerungskonzepte, die auf der permanenten Antizipation der durch Macht, Markt und Geld bestimmten »gesellschaftlichen Interessen « beruhen - keineswegs zu verwechseln mit einem politisch objektivierbaren »gesellschaftlichem Wissenschaftsbedarf« - , öffentlich unter dem Ticket »Mehr Autonomie für die Hochschulen!« zu verhandeln. Dabei steht in allen einschlägigen Lehrbüchern: »Marketing bedeutet als Organisationsprinzip die Außenorientierung « (Sporn 1992, 94). Maximale Autonomie im technischadministrativen Sinne ist allerdings mit maximaler Heteronomie in der inhaltlichen Bestimmung durchaus vereinbar. Aus einem ökonomischen Blickwinkel existiert dieser Widerspruch tatsächlich nicht!

tische Nachfrage steuert das Studienangebot, das sich im Wettbewerb mit anderen Ausbildungsangeboten behaupten muß. Es findet eine ständige Produktdifferenzierung und Anbieterauslese statt, die es im übrigen der Hochschule wirtschaftlich verbietet, den größten Teil der Professoren ..... auf Lebenszeit einzustellen.« 


\section{Hierarchische Differenzierung und standortpolitische Mittelkon- zentration: Die marktförmig recycelten Ordinarien »neuen Typs«}

Jedes Marktmodell suggeriert ideologisch die Gleichheit der Ausgangsbedingungen für die Teilnahme am Wettbewerb. Diese Chancengleichheit ist jedoch an den Hochschulen noch weniger vorhanden als irgendwo sonst in der Gesellschaft. Zusammengefaßt lautet die Kritik am Konzept der »wirtschaftlich denkenden Hochschule«, daß damit die vorhandenen Ungleichheitsverhältnisse und spezifisch patriarchalischen Rekrutierungsmechanismen von Wissenschaftskarrieren eher noch gestärkt - und zugleich politisch entthematisiert werden. Ebenso gefördert werden sektorale hierarchische Differenzierungsprozesse, die sich insbesondere in den letzten 10-15 Jahren als Ausdruck marktorientierter Wissenschaftssteuerung, vor allen Dingen über die Forschungsfördermechanismen, entwickelt haben. Dazu gehört etwa das Ausstattungsgefälle zwischen naturwissenschaftlichtechnischen und sozial- bzw. geisteswissenschaftlichen Bereichen (vgl. Schneekloth 1990, 100f, 113f).

Um ein signifikantes Beispiel zu beleuchten: Das Drittmittelvolumen ist deswegen ein allgemein anerkannter Indikator für die Leistungsfähigkeit von Hochschulen, weil Drittmittel die Kriterien einer Vergabepraxis durch die hochselektierte »scientific community« ( $80 \%$ der Drittmittel werden über die Deutsche Forschungsgemeinschaft vergeben), der Förderung von Spitzenforschung und (in der Verbindung mit Promotionen) der Förderung des sog. wissenschaftlichen Nachwuchses verbinden. Zudem sind Drittmittel infolge der 3. HRG-Novelle (1985) von einem politischen Genehmigungsvorbehalt der Hochschulgremien enthoben. Last not least bedeuten Drittmittel eine Entlastung des öffentlichen Wissenschaftshaushaltes, weswegen es einsehbar ist, daß insbesondere Ministerien Grundausstattungszuweisungen zunehmend gerne anhand des Drittmittelaufkommens $\gg$ differenzieren«. Nominal steigerte sich das Drittmittelvolumen von 630 Millionen (1970) auf 3,35 Milliarden (1993). Signifikant sind die Proportionen: betrugen die Grundausstattungsmittel für Forschung und Lehre 1980 noch das sechsfache, so 1990 das viereinhalbfache des Drittmittelsektors (HRK 1996, 11). Dessen Steuerungsfunktion geht jedoch weit über seine unmittelbare Finanzsumme hinaus. In dem Maße wie Drittmittel als Indikator für »Modernität« und »Leistungsfähigkeit« von Hochschuleinheiten galten, begünstigten sie im Verlauf der 80er Jahre auch einen ungleichen Konzentrationsprozeß der Grundmittel, da die Attraktivität der Fachbereiche für private und öffentliche Forschungsförderer in direkter Relation zur Qualität ihrer Ausstattung steht (Schneekloth 1990, 12I; vgl. auch Weber 1987, 113f). Solche Ungleichgewichte der materiellen Grundausstattung werden nun bei den aktuell gängigen Output-Indikatorisierungen stillschweigend 
vorausgesetzt, da diese, wie etwa in Nordrhein-Westfalen, lediglich »laufende Mittel für Lehre und Forschung«, Output-Größen und »Anzahl der Wissenschaftler« in ein Verhältnis setzen (Hödl 1994, 153). So befürchtet etwa ein Befürworter der Haushaltsautonomie, der Rektor der Gesamthochschule Wuppertal:

$»$ Wenn die Verteilung der Titelgruppe für Lehre und Forschung nach verkürzten Effizienzkriterien vorgenommen wird, werden die damit eingeleiteten Marktprozesse eine Spaltung der Hochschullandschaft zur Folge haben. Die effizienteren Hochschulen werden immer mehr Mittel ... erhalten und auf der Grundlage der bereits überdurchschnittlichen Personal- und Geräteausstattung ihren Vorsprung ausbauen können. Dieser Prozeß verläuft dann progressiv und ohne cinen Umkehrpunkt.« (Hödl ebd., 162)

Wie wahr! Sich darüber zu beschweren ist allerdings müßig, weil die Herstellung einer ungleichen Hochschullandschaft genau das verkündete Ziel einer Marktsteuerung ist.

Durch eine Quantifizierung von Output-Größen wird zunächst eine Erhöhung von Transparenz suggeriert, was in einem vordergründigen Sinne sogar zutreffend ist. Jede auf diese Daten bezogene Verwendung des Effizienzbegriffs, hat allerdings »eine Präzisierung der zugrundeliegenden Ziele zur Voraussetzung « (Haslinger 1994, 166). In dem Maß wie Elemente politischer Steuerung von Hochschulaufgaben, sei es über Parlamente oder »interessenpluralistische« Gremien, in ihrer Zielfindungsfunktion zugunsten von Momenten ökonomischer Selbstregulation relativiert werden, bedeutet dies vor allem eine Stärkung der Gruppen, die traditionell sowieso über wissenschaftliche Abläufe maßgeblich entschieden haben: freigesetzt würden folglich lediglich Mechanismen verstärkter Selbstidentifikation der »scientific community « in ihren vertrauten Paradigmen. So ist erklärbar, daß die gegenwärtig überwiegend gehandelten Output-Indikatoren - quantifizierbare Forschungsleistungen, Zertifikate wie Promotionen und Habilitationen etc. - am traditionellen professoralen Tätigkeitsprofil ansetzen. Die dem zugrundeliegenden informellen Förderungs- und Anerkennungspraxen werden folglich nicht politisch begründet, erst recht nicht zum Gegenstand einer Bewertung gemacht, sondern ebenso stillschweigend vorausgesetzt. Dies ist eher strukturkonservativ als innovativ, da gerade für radikal neue Forschung »jedwede Beurteilungsmaßstäbe « innerhalb der gültigen Paradigmen fehlen (so Haslinger in Anlehnung an Kuhn; ebd., 168). Der Wissenschaftsrat hat dies schon 1985 (S. 32) in seiner Entwurfsfassung der Empfehlungen zum Wettbewerb im deutschen Hochschulsystem - quasi die religiöse »Urschrift« aller nachfolgenden »Wettbewerbspapiere « - offen ausgeplaudert:

»Funktionierender Wettbewerb setzt voraus, daß es anerkannte Instanzen und Verfahren für die Bewertung von Ausbildungs- und Forschungsleistungen gibt. Die Schlüsselbedeutung dieser Instanzen im Wettbewerbsprozeß liegt auf der Hand. Sie sind es, die Leistungen durch die Zuweisung knapper Güter honorieren, durch die also die Leistungsstimuli des Wettbewerbs wirksam werden.« 
Diese göttlichen Schiedsrichter werden dann auch benannt: es sind - wer hätte das gedacht? - sdie Selbstverwaltungsorganisationen der Wissenschaft« (ebd.), also das einschlägig ausgewiesene old boys network der Forschungsförderorganisationen, Fach- und Standesverbände. Folgerichtig bevorzugt der Wissenschaftsrat, mittlerweile von einer ostdeutschen Technikprofessorin angeführt, in seinen jüngsten »Empfehlungen zur Stärkung der Lehre in den Hochschulen durch Evaluation« (19.6.1996) die Mechanismen des »Peer Review《, d.h. das sich gegenseitige Bewerten »interner《 und »externer《 Professoren (zur Kritik vgl. Webler 1996). Die HRK $(1995,9 f)$ hat ähnliche Vorstellungen. Unverkennbar ist der Versuch der wissenschaftspolitischen Spitzenverbände, die aktuelle Konjunktur um »Leistung « und »Evaluation « dafür zu nutzen, jenseits traditioneller politischer Aushandlungsprozeduren zusätzliche exklusive Entscheidungsstränge einer selbst-referentiellen Bewertung der »scientific community « zu installieren - und so die Sache in bewährter Form im Griff zu behalten!

Diese Stärkung von traditionellen Hierarchien widerspricht nicht der Behauptung einer Relativierung pauschaler Standesprivilegien der Professorenschaft. Sie entspricht vielmehr der angestrebten stärkeren funktionalen und vertikalen Differenzierung von Lehr- und Forschungsaufgaben an Hochschulen, bzw. von »Ausbildungs-« und »Wissenschaftsorientierung«, die in ihren Konsequenzen offenbar bis in die Personalstruktur fortgesetzt werden soll. Das Bild einer homogenen Professorenschaft ist ohnehin mittlerweile eher das Ergebnis symbolischer Standespolitik. In der Realität hat die Differenzierung der traditionellen Berufsrolle längst eingesetzt: die ursprüngliche Allzuständigkeit der »Gesamtvertretung « eines Faches zerfasert sich seit längerem in eine ungleiche Gewichtung verschiedener Funktionen, vereinfacht personifiziert in den Typen des reinen Lehrbeamten, des Wissenschaftsmanagers und des Spitzenforschers, der kaum noch einen Hörsaal betritt (Bultmann 1993, 78). Dies eröffnet einerseits Chancen der Professionalisierung wissenschaftlicher Berufstätigkeit auf der Basis einer aufgabenorientierten, d.h. politisch reflektierten Arbeitsteilung, kurz: Abflachung von Hierarchien und Demokratisierung. Das ist jedoch nicht die bestimmende Tendenz. Diese ist vielmehr in Ansätzen einer weiteren hierarchischen Spezialisierung erkennbar. Politische Praxis ist zunächst der Versuch einer weiteren Deregulierung der Beschäftigungsverhältnisse des Mittelbaus. Die Vorschläge des Wissenschaftsrates zur Neustrukturierung der Doktorandenausbildung (1995) laufen im Kern darauf hinaus, künftig die Promotionsphase vorrangig über Stipendien der Stiftungen und der Forschungsförderung zu organisieren - und sie damit erstens von der Hochschulpersonalstruktur zu entkoppeln, um sie zweitens mit den Mechanismen der hochschulexternen Wissenschaftssteuerung effektiv zu verkoppeln. In dem Maße, wie Differenzierungsprozesse zwi- 
schen Ausbildungs- und Forschungsfunktionen der Hochschulen eine Teilung der Hochschullandschaft über das bisherige Maß hinaus zur Folge hätten, würde sich das traditionelle Lehrpersonal entsprechend über eine »Differenzierung« von Deputaten, Besoldungen und Rechten in der Spannweite der tayloristisch zerlegten »Dienstleistungen« wie »Lehre« als Massenveranstaltung und $»$ Forschung « als Elitenrekrutierung aufsplitten. Im institutionellen Schlüsselbegriff der »Dienstleistung « als Leitbild ist die Kundenorientierung eines Verständnisses von Studium als arbeitsmarktbezogene Stoffvermittlung und von »Wissenschaft « als sektoral abgesonderte High-Tech-Forschung bereits angelegt.

Es ist müßig zu ergänzen, daß sich diese Perspektive hierarchischer Spezialisierung nicht einer methodisch reflektierten Arbeitsteilung und wissenschaftspolitisch ausgewiesenen Schwerpunktsetzungen im Hinblick auf einen definierbaren gesellschaftlichen Wissenschaftsbedarf verdankt, sondern einer begriffslosen Verkoppelung mit dem aktuellen gesellschaftlichen Mainstream ökonomisch-technischer »Effizienzsteigerung «.

\section{Zusammenfassende Kritik - und: Wie könnte es anders gehen?}

Die aktuell diskursprägenden Konzepte zur Deregulierung der Hochschule laufen in letzter Konsequenz auf eine Aufhebung der Schwundformen politischer Regulation des Hochschulsektors hinaus, um diese durch ökonomische Selbststeuerungsmechanismen zu ersetzen. Dem liegt eine in der Öffentlichkeit dominante Interpretation der »Hochschulkrise« zugrunde, die sich aus wohlgefälliger »Bürokratismuskritik« speist. Wenn das CHE etwa die Schwerfälligkeit heutiger Hochschulen auf die praktische Konkurrenz unvereinbarer »Leitbilder« der Steuerung entsprechend der »ViererTypologie« (Gelehrtenrepublik, Behörde, Gruppeninstitution, Dienstleistungsbetrieb) Müller-Bölings zurückführt, so entspricht dies zunächst der Wahrheit institutionalisierter politischer Kompromisse der 70er-Jahre. Unterschlagen wird jedoch gleichzeitig der gesellschaftliche Inhalt dieser Kompromisse, der sich in bestimmten, politisch regulierten, Zielsetzungen für das Hochschulsystem (etwa: »soziale Öffnung«, »wissenschaftlichtechnischer Fortschritt« etc.) ausdrückte. ${ }^{13}$ Die Konkurrenz politisch ver-

13 Das bedeutet nicht, daß sich traditionelle wachstumsorientierte Steuerungsformen der 70er Jahre einfach konservieren ließen, sondern daß »Effizienz« grundsätzlich nur über einen gesellschaftsorientierten Interessen- und Politikbezug bestimmbar ist, was bei Müller-Böling u.a. unter den Tisch fällt. Der Ausrichtung der Hochschulfinanzierung an der Studienanfängerzahl beispielsweise liegt keine rein »technische« Entscheidung zu Grunde, sondern eine Kombination sozialstaatlicher mit bildungsökonomisch-planerischen Zielen (»social demand approach «), welche einer rein wirtschaftlichen Bewertung des Bildungsverhaltens entgegensteht. Das $»$ Recht auf Bildung« wird in diesem technokratischen Mechanismus höher taxiert als etwa der "Arbeitsmarktbedarf«. Wer den Finanzierungsmechanismus ändern will, kann folglich nicht nur effizienztechnisch argumentieren, 
einbarter Interessen wird so bereits im Ansatz motivationspsychlogisch auf ein »Kommunikationsproblem《 reduziert, welches durch eine managementförmige Koordination auf »Leitbilder « als aufhebbar erscheint. In dieser Logik erfolgt dann die Reorganisation der Hochschulen nicht mehr auf der Basis politisch transparenter Interessen oder legitimationsfähiger und -bedürftiger gesellschaftlicher Zielsetzungen, sondern im Namen eines sachzwanglogisch neutralisierten (technischen) Effizienzbegriffes. Dies ist eine wirksame Operation der restlosen Entpolitisierung der Hochschulen. Sie vereinbart die Rückkehr zu einer Vorstellung »reiner«, d.h. von Interessen und Politik »unberührter «, Wissenschaft im Sinne des ordinarienzentrierten $»$ Fachvertretungsprinzip« mit der unhinterfragbaren Inhaltslosigkeit ökonomisch-technischer »Professionalität« (vgl. etwa Fischer/Mandell 1994). Diese Komposition sagt auch etwas über die soziale Basis des Konzeptes aus.

Grundlage jedes alternativen Denk- und Handlungsansatzes kann daher nur die Re-Politisierung der Wissenschaft sein. Dies klingt emphatisch, meint aber zunächst nur, daß wissenschaftliche Entwicklungs-, Entscheidungsund Evaluationsprozesse entgegen der dominanten Tendenz ihrer elitärtechnokratischen Abschottung gegenüber der Öffentlichkeit für eine größere Vielfalt von Interessen, Sichtweisen und konkurrierenden Konzepten geöffnet, d.h. »politisiert« werden müssen, auf daß sich die Reproduktion von Wissenschaft perspektivisch stärker über politische Aushandlungsprozesse und öffentliche Legitimation reguliert. Daß diese Repolitisierung nicht möglich ist, auf der Basis der »Fortschrittskoalition « der 70er Jahre und einer bloßen Aktualisierung damaliger Steuerungskompromisse in der Spannweite von Drittelparität und Studienreformkommissionen dürfte offenkundig sein; eine Feststellung, die nicht der Verteidigung und Erweiterung etwa sozialstaatlicher Steuerungselemente in der Bildungs- und Hochschulplanung widerspricht. Operativer Orientierungspunkt einer Erneuerung der Hochschulen kann nicht ein zukünftiger gesellschaftlicher Zustand, sondern zunächst »nur« die alltägliche Produktion der »ökologischen Krise« durch die Mechanismen des wissenschaftlichen Normalbetriebs sein, einschließlich der durch ihn geförderten ideologischen Problementsorgung und -verdrängung. Theoretisch und praktisch angedacht ist dies etwa im Ansatz der »ökologischen Allianzen« (Becker/Wehling 1993, 77f; Nitsch 1995), der schrittweisen Entwicklung neuer Beteiligungsformen (Nitsch 1991) oder in Entwürfen anti-technokratischer und antihegemonialer Strukturen des Wissenschaftstranfers (Dehler 1990). Über die defensive Situation alternativer Akteure sollten keine Zweifel aufkom-

sondern müßte politisch offenlegen, welchen Bildungsbegriff er hat bzw. wie er zu diesen ursprünglichen Zielsetzungen steht. 
men: aus hochschulinterner Perspektive wäre dies zunächst eine Praxis des Sand-Ins-Getriebe-Streuens oder der schrittweisen Institutionalisierung von Kritik »mit dem Ziel, innerwissenschaftliche Wahrnehmungsmuster und Arbeitsformen zu verändern, sie für soziale und ökologische Problemlagen zu öffnen« (Becker/Wehling ebd., 23). Dabei wäre aktuell schon viel gewonnen, wenn sich eine Gegenöffentlichkeit zur Schein-Evidenz ökonomisch-technokratischer Rationalitätskriterien, mit denen aktuell der Umbau der Hochschulen in Angriff genommen wird, ausbilden würde. Eine Politisierung von Effizienzkriterien führt allein insofern in die erforderliche Richtung, als sich etwa anhand der Personal- und Entscheidungssstrukturen plastisch zeigen läßt, daß das wirkliche Rationalitätsdefizit der Hochschulen ein Demokratie- und Politikdefizit ist.

\section{Literatur}

Becker, Egon; Jungblut, Gerd (1972): Strategien der Bildungsprodukiton, Frankfurt.

Becker, Egon; Wehling, Peter (1993): Risiko Wissenschaft - ökologische Perspektiven in Wissenschaft und Hochschule, Frankfurt.

Behrens, Thomas (1996): Globalhaushalt und Personalpolitik an den Hochschulen, in: Das Hochschulwesen 1996/2, S.121-127.

Block, Hans-Jürgen (1994): Magister en masse - Die Philosophische Fakultät, wenn ein Fünftel des Jahrgangs zur Universität geht, in: Bayerisches Staatsinstitut für Hochschulforschung und Hochschulplanung (Hrsg.), Beiträge zur Hochschulforschung 2-1994, S. 215 226.

Bochow, Michael; Joas, Hans (1987): Wissenschaft und Karriere - Der berufliche Verbleib des akademischen Mittelbaus, Frankfurt.

Bultmann, Torsten; Weitkamp, Rolf (1995): Ideologische Realitätskonstruktion - Zur Diskussion um »Studierfähigkeit« und Selektion, in: Forum Wissenschaft 3/1995.

Bultmann, Torsten (1993): Zwischen Humboldt und Standort Deutschland - Die Hochschulpolitik am Wendepunkt, Marburg.

Bundesministerium für Bildung, Wissenschaft, Forschung und Technologie (BMBF 1995): Grund-und Strukturdaten 1995/96, Bonn.

- (BMBF 1996): Bundesbericht Forschung 1996 (Bundestags-Drucksache 13/4554), Bonn.

Bundesvertretung akademischer Mittelbau (BAM 1991): Wissenschaft als Beruf - Forderungen an eine sachgerechte Personalstruktur (Funktionsstellen), in: BdWi (Hrsg.), Hochschulstrukturen - Studium und Lehre in den 90er Jahren (Fortum Wissenschaft Studienhefte 16), Bonn und Marburg.

Daxner, Michael (1991): Entstaatlichung und Veröffentlichung - Die Hochschule als republikanischer Ort, Köln.

- (1993): Die Wiederherstellung der Hochschule, Köln.

- (1993a): Globalhaushalt - Mythos und Realität, in: Bayerisches Staatsinstitut für Hochschulforschung und Hochschulplanung (Hrsg.), Beiträge zur Hochschulforschung 2-1993, S.111134.

Daxner, Michael; Schindler, Götz (1993): Studienzeitverkürzung, in: Das Hochschulwesen 1993/4, S.177-180.

Dehler, Joseph (1990): Wider die Verschwendung von Wissen - Vernetzung in Regionalen Wissenschaftszentren, Frankfurt.

»Eckwertepapier« der Bund-Länder-Arbeitsgruppe zur Vorbereitung des bildungspolitischen Spitzengesprächs (I993), dokumentiert in: Das Hochschulwesen 1993/4, S.166-175.

Enders, Jürgen (1989), Beschäfitgungssituation im Akademischen Mittelbau, Projektbericht, Kassel. 
- (1995): Die Hochschullehrer in der Massenuniversität - eine Expertise im Auftrag der GEW, Kassel.

Fischer, Frank; Mandell, Alan (1994): Bildungspolitik und die postindustrielle Transformation: »Excellence« als technokratische Ideologie, in: Sünker, Heinz; Timmermann, Dieter; Kolbe, Fritz-Ulrich (Hrsg), Bildung, Gesellschaft, soziale Ungleichheit - Internationale Beiträge zur Bildungssoziologie und Bildungstheorie, Frankfurt 1994, S.120-144.

Franke, Siegfried F. (1994): Bildungspolitik in der Demokratie, in: Grözinger, Gerd; Hödl, Erich (Hrsg.), Hochschulen im Niedergang - Zur Politischen Ökonomie von Forschung und Lehre, Marburg, S.33-68.

Fried, Barbara; Kaindl, Christina; Markard, Morus (1995): Psychologie an der FU Berlin: Modell für die Einsparung bzw. Entsorgung kritischer Wissenschaft, in: Forum Kritische Psychologie 35, S. 156-172.

Gewerkschaft Erziehung und Wissenschaft (GEW 1994; 1995): Datenservice: Wissenschaft '94; Datenservice Wissenschaft' '95, Frankfurt.

Hartung, Dirk (1993): Studium und Beruf, in: Hilde Schramm (Hrsg. im Auftrag der GEW), Hochschule im Umbruch, Berlin, S.406-413.

Haslinger, Franz (1994): Effizienzvergleiche als Grundlage der Hochschulpolitik. Einige kritische Anmerkungen, in: Grözinger, Gerd; Hödl, Erich (Hrsg.), Hochschulen im Niedergang - Zur Politischen Ökonomie von Forschung und Lehre, Marburg, S. 165-172.

Höd], Erich (1994): Ökonomische Anreizsysteme zur Hochschulsteuerung, in: Grözinger, Gerd; Hödl, Erich (Hrsg.), Hochschulen im Niedergang - Zur Politischen Ökonomie von Forschung und Lehre, Marburg, S.147-164.

Hochschulrektorenkonferenz (1995): Zur Evaluation im Hochschulbereich unter besonderer Berücksichtigung der Lehre (Entschließung des 176. Plenums vom 3.7.1995), Ms.

- (1996): Zur Finanzierung der Hochschulen (Entschließung des I79. Plenums am 8.19.7.1996), Ms..

Kronberger Kreis (1993): Zur Reform der Hochschule (Schriftenreihe des Frankfurter Instituts für wirtschaftspolitische Forschung e.V. Band 27), Bad Homburg.

Meyer, Hans Joachim (1996): Künftige Anforderungen an Verfassung und Struktur der Hochschulen insbesondere zur Lösung der Finanzprobleme (Vortrag an der TU Dresden 12.6.96), Ms..

Mittelstraß, Jürgen (1992): Die Zukunft der Wissenschaft und die Gegenwait der Universität, in: Stifterverband für die Deutsche Wissenschaft - Mitgliederversammlung 1992 (Ansprachen und Vorträge), Essen.

Ministerium für Wissenschaft und Forschung in NRW (MWuF NRW 1995): Weniger Staat für die staatlichen Hochschulen - Bericht des Gesprächskreises Funktionalreform, Düsseldorf.

Müller-Böling, Detlef (1994): Von der Gelehrtenrepublik zum Diensleistungsunternehmen?, in: Forschung \& Lehre 7/I994, S.272-275.

- (1995): Qualitätssicherung in Hochschulen - Grundlage einer wissenschaftsbasierten Gesellschaft (Begrüßungsansprache zur Eröffnung des CHE am 25.1.1995 in Gütersloh), Ms..

- (1995a): Deutscher Studienfonds zur Qualitätssicherung der Hochschulen - Argumente für und wider einen Beitrag der Siudierenden zur Finanzierung des Hochschulsystems, CHEArbeitspapier Nr.8 (Oktober 1995), Gütersloh.

Nitsch, Wolfgang (1991): Runde Tische an Hochschulen - neue Beteiligungsformen im Wissenschaftsbereich, Frankfurt.

- (1995): Förderung sozial-ökologischer Transformationskeme, in: Fontum Wissenschaft 1/1995, S.80-81.

Nitsch, Wolfgang; Gerhardt, Uta; Offe, Claus; Preuß, K. Ulrich (1965): Hochschule in der. Demokratie, Berlin/Neuwied.

Plander, Harro (1986): Arbeitsplatz Hochschule, Freiburg.

Preißer, Rüdiger (1994): Die Außeralltäglichkeit der deutschen Hochschulen: Hochschule als paradoxe Bürokratie, in: Bayerisches Staatsinstitut für Hochschulforschung und Hochschulplanung (Hrsg.), Beiträge zur Hochschulforschung 2-1994, S. 151-186.

Reuhl, Günter (1992): Wissenschaftskonkurrenz - Hochschulorganisation in den USA, Europa und der Sowjetunion, Frankfurt. 
Schneekloth, Ulrich (1990): Hochschulen zwischen Funktionswandel und Krise, Stuttgart.

Schimank, Uwe (1994): Immer weniger Zeit für die Forschung? - Einige Daten und Überlegungen zur Lage der Professoren an den westdeutschen wissenschaftlichen Hochschulen, in: Bayerisches Staatsinstitut für Hochschulforschung und Hochschulplanung (Hrsg, ), Beiträge zur Hochschulforschung 4-1994, S.685-706.

Sporn, Barbara (1992): Universitätskultur - Ausgangspunkt für eine strategische MarketingPlanung an Universitäten, Heidelberg.

Stifterverband für die Deutsche Wissenschaft (Stifterverband 1994): Hochschulreform durch Leistungswettbewerb und Privatisierung? - Dokumentation eines Symposiums, Essen.

Teichler, Ulrich (1990): Europäische Hochschulsysteme: Die Beharrlichkeit vielfältiger Modelle, Frankfurt.

Weber, Michael (1987): Krise und Zukunft der Hochschulen - eine Denkschrift des BdWi, Marburg.

Webler, Wolf-Dietrich (1996): Stellungnahme zu den »Empfehlungen zur Stärkung der Lehre in den Hochschulen durch Evaluation « des Wissenschaftsrates vom 19.1.1996, in: das Hochschulwesen 1996/3, S.153-155.

Wissenschaftstat (1985): Empfehlungen zum Wettbewerb im deutschen Hochschulsystem Entwurf(Drs. 6736/85), Köln.

- (1993): 10 Thesen zur Hochschulpolitik, Köln.

- (1995): Grunddaten zum Personalbestand der Hochschulen, Köln.

- (1995a): Empfehlungen zur Neustrukturierung der Doktorandenausbildung und -forderung, Köln.

Zeitungen:

TU-Intern: Zeitung der TU Berlin.

Uni-HH: Zeitung der Universität Hamburg.

Uni-Info: Zeitung der Carl von Ossietzky-Universität Oldenburg.

Forschung \& Lehre: Mitteilungen des Deutschen Hochschulverbandes. 